\title{
Weakly Discriminating Vertex-Degree-Based Topological Indices
}

\author{
Juan Rada ${ }^{1}$, José M. Rodríguez ${ }^{2}$, José M. Sigarreta ${ }^{3}$ \\ ${ }^{1}$ Instituto de Matemáticas, Universidad de Antioquia, Medellín, Colombia \\ pablo.rada@udea.edu.co \\ ${ }^{2}$ Departamento de Matemáticas, Universidad Carlos III de Madrid, \\ Madrid, Spain \\ jomaro@math.uc3m.es \\ ${ }^{3}$ Facultad de Matemáticas, Universidad Autónoma de Guerrero \\ Acalpulco, Mexico \\ jsmathguerrero@gmail.com
}

(Received May 20, 2021)

\begin{abstract}
Let $\mathcal{G}_{n}$ be the set of graphs with $n$ vertices and $\mathcal{H} \subseteq \mathcal{G}_{n}$. For each $H \in \mathcal{H}$, let $m(H)=\left\{m_{i, j}(H)\right\}$, where $m_{i, j}(H)$ is the number of edges in $H$ that join a vertex of degree $i$ with a vertex of degree $j$. A vertex-degree-based (VDB, for short) topological index $\varphi$ is discriminating over $\mathcal{H}$ if non-isomorphic graphs in $\mathcal{H}$ have different values of $\varphi$. We say that $\varphi$ is weakly discriminating over $\mathcal{H}$ if the following weaker condition is satisfied for every $H, H^{\prime} \in \mathcal{H}$ :

$$
\varphi(H)=\varphi\left(H^{\prime}\right) \Longleftrightarrow m(H)=m\left(H^{\prime}\right) .
$$

Let $\mathcal{C} \mathcal{T}_{n}$ be the set of chemical trees with $n$ vertices. In this paper we show that many of the well-known VDB topological indices are not weakly discriminating over $\mathcal{C} \mathcal{T}_{n}$. However, the recently introduced Sombor index is weakly discriminating over $\mathcal{C} \mathcal{T}_{n}$. Also, we give conditions under which a $\operatorname{VDB}$ topological index $\varphi$ is weakly discriminating over an arbitrary class $\mathcal{H} \subseteq \mathcal{G}_{n}$.
\end{abstract}




\section{Introduction}

Throughout this paper a graph $G=(V, E)$ is simple, undirected and unweighted, where $V$ is the set of vertices and $E$ the set of edges. If there is an edge from vertex $u$ to vertex $v$ we indicate this by writing $u v$ (or $v u$ ). The degree of the vertex $v$ of $G$ is denoted by $d_{v}$. A vertex $v$ is isolated if $d_{v}=0$. We denote by $n_{i}=n_{i}(G)$ the number of vertices of $G$ with degree $i$ and $m_{i, j}=m_{i, j}(G)$ the number of edges in $G$ joining vertices of degree $i$ and $j$.

Topological indices are molecular descriptors which play an important role in theoretical chemistry, especially in QSPR/QSAR research [22,23]. One important class of topological indices are the so-called vertex-degree based (VDB, for short) topological indices (see [10,18], and for recent results $[1,3,5-7,15-17,24])$.

Let $\mathcal{G}_{n}$ be the set of graphs with $n$ non-isolated vertices and $\mathcal{H} \subseteq \mathcal{G}_{n}$. Let $\Delta=$ $\max \{\Delta(H): H \in \mathcal{H}\}$. Consider the set

$$
K=\{(i, j) \in \mathbb{N} \times \mathbb{N}: 1 \leq i \leq j \leq \Delta\}
$$

lexicographically ordered. Let $h=|K|=\frac{\Delta(\Delta+1)}{2}$. Define the function $m: \mathcal{H} \longrightarrow \mathbb{R}^{h}$ by $m(H)=\left(m_{i, j}(H)\right)_{(i, j) \in K}$, where $H \in \mathcal{H}$. Formally, a VDB topological index is a function $T_{\varphi}: \mathcal{H} \longrightarrow \mathbb{R}$ induced by a vector $\varphi \in \mathbb{R}^{h}$, defined for $H \in \mathcal{H}$ as

$$
T_{\varphi}(H)=m(H) \cdot \varphi
$$

where ". denotes the dot product over $\mathbb{R}^{h}$. For brevity, we simply write

$$
\varphi(H)=m(H) \cdot \varphi
$$

and say that $\varphi$ is a VDB topological index defined over $\mathcal{H}$. Most of the well-known VDB topological indices studied in mathematical chemistry are induced by symmetric functions of the form $\varphi(x, y)$. In other words, the vector $\varphi \in \mathbb{R}^{h}$ has components $(\varphi(x, y))_{(x, y) \in K}$, where $\varphi(x, y)$ is a symmetric function.

From expression (2), we easily deduce that if $H, H^{\prime} \in \mathcal{H}$, then [19]

$$
\varphi(H)=\varphi\left(H^{\prime}\right) \Longleftrightarrow\left[m(H)-m\left(H^{\prime}\right)\right] \cdot \varphi=0 \Longleftrightarrow\left[m(H)-m\left(H^{\prime}\right)\right] \perp \varphi .
$$

For the well-known VDB topological indices $\varphi \in \mathbb{R}^{h}$, the perpendicularity condition above occurs very frequently in most of the important classes of sets $\mathcal{H}$ studied (connected 
graphs, trees, chemical graphs, etc ...). In other words, non-isomorphic graphs of $\mathcal{H}$ have equal value $\varphi$. This degeneracy property has been investigated extensively in mathematical chemistry $[8,9,13]$.

In [19], the concept of discrimination of a VDB topological index was generalized to equivalence relations defined in $\mathcal{H}$. Specifically, if $\sim$ is an equivalence relation defined in $\mathcal{H}$, then $\varphi$ discriminates on $\mathcal{H}$ with respect to $\sim$ if the following condition holds: for all $H, H^{\prime} \in \mathcal{H}$,

$$
\varphi(H)=\varphi\left(H^{\prime}\right) \Longleftrightarrow H \sim H^{\prime}
$$

In this case we say that $\varphi$ is discriminating over $\mathcal{H}$ with respect to the equivalence relation $\sim$. When we consider the isomorphism relation $\simeq$ on $\mathcal{H}$, then we recover the usual concept of discrimination. But there are other interesting equivalence relations, for example, in [19, Example 2.2] we consider the equivalence relation defined on $\mathcal{H}$,

$$
H \sim H^{\prime} \Longleftrightarrow m(H)=m\left(H^{\prime}\right)
$$

If a vertex-degree-based topological index $\varphi$ is discriminating over $\mathcal{H}$ with respect to the relation (3), then we simply say that $\varphi$ is weakly discriminating over $\mathcal{H}$. Since

$$
H \simeq H^{\prime} \Longrightarrow m(H)=m\left(H^{\prime}\right)
$$

it follows that if $\varphi$ is discriminating over $\mathcal{H}$ (in the usual way), then $\varphi$ is weakly discriminating over $\mathcal{H}$.

Naturally a question arises: which VDB topological indices are weakly discriminating over $\mathcal{H}$ ? In Section 2 we study the case $\mathcal{H}=\mathcal{C} \mathcal{T}_{n}$, the set of chemical trees with $n$ vertices. Specifically, we show in Examples 2.1 and 2.2 several well-known VDB topological indices which are not weakly discriminating over $\mathcal{C T}_{n}$ : the Randić index [20], the first Zagreb index [12], the sum-connectivity index [25], the harmonic index [26], first hyper-Zagreb index [21], and the reciprocal sum-connectivity index [15]. On the other hand, we show in Theorem 2.3, that the recently introduced Sombor index [11] is weakly discriminating over $\mathcal{C} \mathcal{T}_{n}$.

In Section 3 we give conditions under which a $\operatorname{VDB}$ topological index $\varphi$ is weakly discriminating over an arbitrary class $\mathcal{H} \subseteq \mathcal{G}_{n}$. Concretely, we show that if the components $\left\{\varphi_{i, j}\right\}_{(i, j) \in K}$ of $\varphi$ are linearly independent over the set of integers $\mathbb{Z}$, then $\varphi$ is weakly discriminating over $\mathcal{H}$. As a byproduct, we show that if the components $\left\{\varphi_{i, j}\right\}_{(i, j) \in K}$ are distinct algebraic numbers, then the exponential $e^{\varphi}$ is weakly discriminating over $\mathcal{H}$. 


\section{Weakly discriminating VDB topological indices over $\mathcal{C} \mathcal{T}_{n}$}

Which VDB topological indices are weakly discriminating over $\mathcal{H}$ ? Let us see some examples when $\mathcal{H}=\mathcal{C} \mathcal{T}_{n}$, the set of chemical trees with $n$ vertices. In this case

$$
K=\{(i, j) \in \mathbb{N} \times \mathbb{N}: 1 \leq i \leq j \leq 4\},
$$

and since $m_{1,1}(T)=0$ for all $T \in \mathcal{C} \mathcal{T}_{n}$ when $n \geq 3$, all VDB topological indices $\varphi$ are vectors in $\mathbb{R}^{9}$ :

$$
\varphi=\left(\varphi_{1,2}, \varphi_{1,3}, \varphi_{1,4}, \varphi_{2,2}, \varphi_{2,3}, \varphi_{2,4}, \varphi_{3,3}, \varphi_{3,4}, \varphi_{4,4}\right)
$$

Example 2.1 The Randić index is induced by the symmetric function $\varphi(x, y)=\frac{1}{\sqrt{x y}}$, so its associated vector is

$$
\varphi=\left(\frac{1}{\sqrt{2}}, \frac{1}{\sqrt{3}}, \frac{1}{2}, \frac{1}{2}, \frac{1}{\sqrt{6}}, \frac{1}{\sqrt{8}}, \frac{1}{3}, \frac{1}{\sqrt{12}}, \frac{1}{4}\right) .
$$

It turns out that the Randić index is not weakly discriminating over $\mathcal{C} \mathcal{T}_{n}$. In fact, consider the trees $S$ and $T$ depicted in Figure 1. Let $p$ be a positive integer. In the tree $S$ there are $4 p+1$ vertices of degree 4 . In the tree $T$ there are $3 p+1$ vertices of degree 4 , there is a path with $p \geq 1$ edges between vertices $u$ and $v$, and there is a path with $p$ edges between vertices $w$ and $z$. By a counting argument, one easily sees that $S$ and $T$ are trees with $20 p+9$ vertices. Moreover,

$$
m_{1,2}(S)=8 p+4, \quad m_{2,4}(S)=8 p+4, \quad m_{4,4}(S)=4 p,
$$

and $m_{i, j}(S)=0$ for all other values of $i, j$. On the other hand,

$$
m_{1,2}(T)=6 p+4, \quad m_{2,2}(T)=2 p, \quad m_{2,4}(T)=12 p+4,
$$

and $m_{i, j}(T)=0$ for all other values of $i, j$. In particular, $m(S) \neq m(T)$. However,

$$
\varphi(S)=\varphi(T)=(6 \sqrt{2}+1) p+3 \sqrt{2}
$$

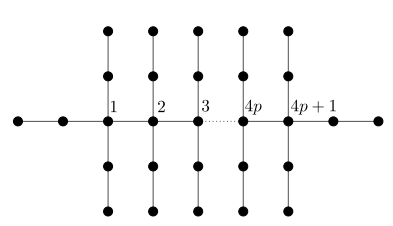

$S$

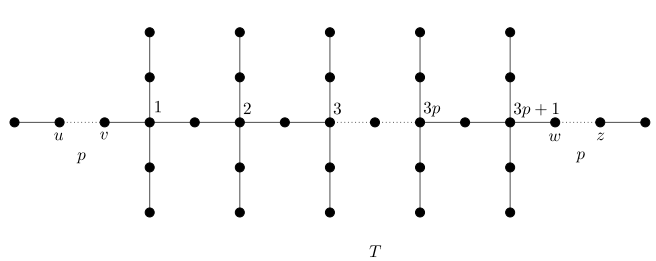

Figure 1. Trees with equal Randić index but different $m$-structure. 
Example 2.2 Consider the following VDB topological indices induced by the function $\varphi(x, y)$ :

\begin{tabular}{|c|c|}
\hline$\varphi(x, y)$ & Name \\
\hline$x+y$ & first Zagreb index \\
\hline$(x+y)^{2}$ & first hyper-Zagreb index \\
\hline$\frac{1}{\sqrt{x+y}}$ & sum-connectivity index \\
\hline$\sqrt{x+y}$ & reciprocal sum-connectivity index \\
\hline$\frac{2}{x+y}$ & harmonic index \\
\hline
\end{tabular}

All these VDB topological indices have the following property in common:

$$
\varphi(1,3)=\varphi(2,2) ; \quad \varphi(1,4)=\varphi(2,3) ; \quad \varphi(2,4)=\varphi(3,3)
$$

Hence, for every $U \in \mathcal{C} \mathcal{T}_{n}$,

$$
\begin{aligned}
\varphi(U)= & \varphi(1,2) m_{1,2}(U)+\varphi(1,3)\left(m_{1,3}(U)+m_{2,2}(U)\right)+ \\
& \varphi(1,4)\left(m_{1,4}(U)+m_{2,3}(U)\right)+\varphi(2,4)\left(m_{2,4}(U)+m_{3,3}(U)\right)+ \\
& \varphi(3,4) m_{3,4}(U)+\varphi(4,4) m_{4,4}(U) .
\end{aligned}
$$

So a condition that implies that two trees $S, T \in \mathcal{C} \mathcal{T}_{n}$ have equal value of $\varphi$ is the following:

$$
\begin{gathered}
m_{1,3}(S)+m_{2,2}(S)=m_{1,3}(T)+m_{2,2}(T) \\
m_{1,4}(S)+m_{2,3}(S)=m_{1,4}(T)+m_{2,3}(T) \\
m_{2,4}(S)+m_{3,3}(S)=m_{2,4}(T)+m_{3,3}(T) \\
m_{i, j}(S)=m_{i, j}(T) \text { for the other values of }(i, j) \in K .
\end{gathered}
$$

Consider the trees $S, T \in \mathcal{C} \mathcal{T}_{n}$ shown in Figure 2. There is a path with $p \geq 1$ edges between vertices $u$ and $v$ in the tree $S$, and there is a path with $p+6$ edges between vertices $w$ and $z$ in the tree T. Note that

$$
\begin{array}{llll}
m_{1,2}(S)=2 ; & m_{1,3}(S)=6 ; & m_{1,4}(S)=0 ; & m_{2,2}(S)=p ; \quad m_{2,3}(S)=4 ; \\
m_{2,4}(S)=0 ; & m_{3,3}(S)=4 ; & m_{3,4}(S)=0 ; & m_{4,4}(S)=0
\end{array}
$$

and

$$
\begin{array}{llll}
m_{1,2}(T)=2 ; & m_{1,3}(T)=0 ; & m_{1,4}(T)=4 ; & m_{2,2}(T)=p+6 ; \quad m_{2,3}(T)=0 ; \\
m_{2,4}(T)=4 ; & m_{3,3}(T)=0 ; \quad m_{3,4}(T)=0 ; \quad m_{4,4}(T)=0 .
\end{array}
$$

Clearly, conditions (5) hold so $\varphi(S)=\varphi(T)$. However, $m(S) \neq m(T)$, which implies that none of these VDB topological indices are weakly discriminating over $\mathcal{C T}_{n}$. 

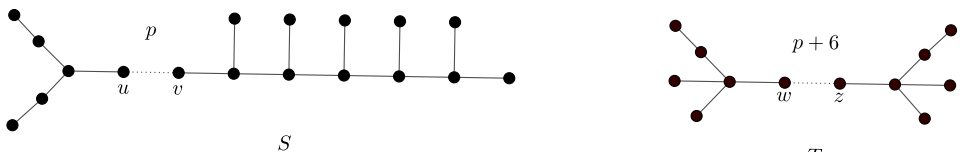

$T$

Figure 2. Trees with equal $\varphi$ but different $m$-structure.

Hence, many of the well known VDB topological indices are not weakly discriminating over $\mathcal{C} \mathcal{T}_{n}$, as we have seen in the previous examples. However, the Sombor index is weakly discriminating over $\mathcal{C} \mathcal{T}_{n}$, as we shall see in our next result. Recall that the Sombor index is induced by the symmetric function $S O(x, y)=\sqrt{x^{2}+y^{2}}$, so the $S O$ vector is given by

$$
S O=(\sqrt{5}, \sqrt{10}, \sqrt{17}, 2 \sqrt{2}, \sqrt{13}, 2 \sqrt{5}, 3 \sqrt{2}, 5,4 \sqrt{2}) .
$$

If $T$ is a chemical tree with $n$ vertices, then

$$
\begin{aligned}
S O(T)= & \sqrt{5}\left(m_{1,2}+2 m_{2,4}\right)+\sqrt{2}\left(2 m_{2,2}+3 m_{3,3}+4 m_{4,4}\right) \\
& +\sqrt{10} m_{1,3}+\sqrt{17} m_{1,4}+\sqrt{13} m_{2,3}+5 m_{3,4} .
\end{aligned}
$$

We will also use the following well-known relations:

$$
\begin{aligned}
& 2 m_{1,1}+m_{1,2}+m_{1,3}+m_{1,4}=n_{1} \\
& m_{1,2}+2 m_{2,2}+m_{2,3}+m_{2,4}=2 n_{2} \\
& m_{1,3}+m_{2,3}+2 m_{3,3}+m_{3,4}=3 n_{3} \\
& m_{1,4}+m_{2,4}+m_{3,4}+2 m_{4,4}=4 n_{4}
\end{aligned}
$$

Theorem 2.3 The Sombor index SO is weakly discriminating over $\mathcal{C} \mathcal{T}_{n}$.

Proof. Let $T \in \mathcal{C} \mathcal{T}_{n}$ and set $m_{i, j}=m_{i, j}(T)$ and $n_{i}=n_{i}(T)$. By (8),

$$
\begin{aligned}
12 n= & 12\left(n_{1}+n_{2}+n_{3}+n_{4}\right) \\
= & 18 p+6 q-10 m_{3,3}-18 m_{4,4}-27 m_{2,4} \\
& +16 m_{1,3}+15 m_{1,4}+10 m_{2,3}+7 m_{3,4},
\end{aligned}
$$

where

$$
p=m_{1,2}+2 m_{2,4}
$$


and

$$
q=2 m_{2,2}+3 m_{3,3}+4 m_{4,4}
$$

Assume now that $T^{\prime} \in \mathcal{C} \mathcal{T}_{n}$ is such that $S O(T)=S O\left(T^{\prime}\right)$. Set $m_{i, j}^{\prime}=m_{i, j}\left(T^{\prime}\right)$ and $n_{i}^{\prime}=n_{i}\left(T^{\prime}\right)$. Since

$$
\{5, \sqrt{2}, \sqrt{5}, \sqrt{10}, \sqrt{13}, \sqrt{17}\}
$$

is a linearly independent set over $\mathbb{Z}$, it follows by (7) that

$$
\begin{aligned}
& p=m_{1,2}^{\prime}+2 m_{2,4}^{\prime} \\
& q=2 m_{2,2}^{\prime}+3 m_{3,3}^{\prime}+4 m_{4,4}^{\prime}
\end{aligned}
$$

and

$$
\begin{aligned}
& m_{1,3}=m_{1,3}^{\prime}, \quad m_{1,4}=m_{1,4}^{\prime} \\
& m_{2,3}=m_{2,3}^{\prime}, \quad m_{3,4}=m_{3,4}^{\prime} .
\end{aligned}
$$

Again by (8), (12), (13), and (14),

$$
\begin{aligned}
12 n= & 12\left(n_{1}^{\prime}+n_{2}^{\prime}+n_{3}^{\prime}+n_{4}^{\prime}\right) \\
= & 18 p+6 q-10 m_{3,3}^{\prime}-18 m_{4,4}^{\prime}-27 m_{2,4}^{\prime} \\
& +16 m_{1,3}+15 m_{1,4}+10 m_{2,3}+7 m_{3,4} .
\end{aligned}
$$

Now from relations (9) and (15) we conclude that

$$
10\left(m_{3,3}-m_{3,3}^{\prime}\right)+18\left(m_{4,4}-m_{4,4}^{\prime}\right)+27\left(m_{2,4}-m_{2,4}^{\prime}\right)=0 .
$$

The general solution of the Diophantine equation

$$
10 x+18 y+27 z=0
$$

is

$$
\left.\begin{array}{c}
x=-54+54 k \\
y=27-27 k \\
z=2-2 k
\end{array}\right\},
$$

where $k \in \mathbb{Z}$. Hence, from (16) we deduce

$$
\begin{aligned}
& m_{3,3}-m_{3,3}^{\prime}=-54+54 k, \\
& m_{4,4}-m_{4,4}^{\prime}=27-27 k, \\
& m_{2,4}-m_{2,4}^{\prime}=2-2 k,
\end{aligned}
$$


for some $k \in \mathbb{Z}$. On the other hand, from (11), (13), and (17),

$$
\begin{aligned}
2\left(m_{22}-m_{22}^{\prime}\right) & =3\left(m_{3,3}^{\prime}-m_{3,3}\right)+4\left(m_{4,4}^{\prime}-m_{4,4}\right) \\
& =3(54-54 k)+4(-27+27 k) .
\end{aligned}
$$

Hence,

$$
m_{2,2}-m_{2,2}^{\prime}=27-27 k \text {. }
$$

Now by (10), (12), and (17),

$$
m_{1,2}-m_{1,2}^{\prime}=2\left(m_{2,4}^{\prime}-m_{2,4}\right)=2(2 k-2) .
$$

Consequently,

$$
m_{1,2}-m_{1,2}^{\prime}=4 k-4
$$

From (14),

$$
\begin{aligned}
& m_{1,3}-m_{1,3}^{\prime}=0 \\
& m_{1,4}-m_{1,4}^{\prime}=0 \\
& m_{2,3}-m_{2,3}^{\prime}=0 \\
& m_{3,4}-m_{3,4}^{\prime}=0 .
\end{aligned}
$$

Adding the left sides and right sides of relations (18)-(26), we obtain

$$
\begin{aligned}
\sum_{1 \leq i \leq j \leq 4} m_{i, j}-\sum_{1 \leq i \leq j \leq 4} m_{i, j}^{\prime} & =(-54+54 k)+(27-27 k)+(2-2 k) \\
+(27-27 k)+(4 k-4) & =2 k-2 .
\end{aligned}
$$

Since

$$
n-1=\sum_{1 \leq i \leq j \leq 4} m_{i, j}=\sum_{1 \leq i \leq j \leq 4} m_{i, j}^{\prime}
$$

it follows from (27) that $k=1$. Thus, from relations (18)-(22),

$$
\begin{array}{ll}
m_{3,3}=m_{3,3}^{\prime} & m_{4,4}=m_{4,4}^{\prime} \quad m_{2,4}=m_{2,4}^{\prime} \\
m_{2,2}=m_{2,2}^{\prime} & m_{1,2}=m_{1,2}^{\prime} .
\end{array}
$$

Finally, from (14) and (28),

$$
m(T)=m\left(T^{\prime}\right)
$$




\section{Conditions which assure the weakly discrimination property on an arbitrary class}

In our next result we give conditions on a VDB topological index which assures the weakly discrimination property over an arbitrary class $\mathcal{H} \subseteq \mathcal{G}_{n}$.

Theorem 3.1 Let $\varphi \in \mathbb{R}^{h}$ be a VDB topological index defined over $\mathcal{H} \subseteq \mathcal{G}_{n}$ such that $\left\{\varphi_{i, j}\right\}_{(i, j) \in K}$ is a linearly independent set over $\mathbb{Z}$, the set of integers. Then $\varphi$ is weakly discriminating over $\mathcal{H}$.

Proof. Let $H, H^{\prime} \in \mathcal{H}$ and assume that $\varphi(H)=\varphi\left(H^{\prime}\right)$. Then

$$
0=\left(m(H)-m\left(H^{\prime}\right)\right) \cdot \varphi=\sum_{(i, j) \in K}\left(m_{i, j}(H)-m_{i, j}\left(H^{\prime}\right)\right) \varphi_{i, j} .
$$

Since $m_{i, j}(H)-m_{i, j}\left(H^{\prime}\right) \in \mathbb{Z}$ for all $(i, j) \in K$ and $\left\{\varphi_{i, j}\right\}_{(i, j) \in K}$ is a linearly independent set over $\mathbb{Z}, m_{i, j}(H)-m_{i, j}\left(H^{\prime}\right)=0$ for all $(i, j) \in K$. Consequently, $m(H)=m\left(H^{\prime}\right)$.

Remark 3.2 The converse of Theorem 3.1 does not hold. For instance, the Sombor index is weakly discriminating over $\mathcal{C} \mathcal{T}_{n}$. However, the components $\mathrm{SO}_{2,2}, \mathrm{SO}_{3,3}$, and $\mathrm{SO}_{4,4}$ are $2 \sqrt{2}, 3 \sqrt{2}$, and $4 \sqrt{2}$, respectively. Note that

$$
3 \mathrm{SO}_{2,2}+2 \mathrm{SO}_{3,3}+(-3) \mathrm{SO}_{4,4}=0
$$

so the components of $S O$ are not linearly independent over $\mathbb{Z}$.

Besicovitch's Theorem can be used to construct many examples of VDB topological indices that are weakly discriminating over $\mathcal{H} \subseteq \mathcal{G}_{n}$.

Theorem 3.3 [4] (Besicovitch) Let $n>1$ be any integer and $p_{1}, \ldots, p_{k}$ distinct positive prime numbers. The set of $n^{k}$ radicals,

$$
\left\{\sqrt[n]{p_{1}^{m(1)} p_{2}^{m(2)} \cdots p_{k}^{m(k)}}: 0 \leq m(i)<n, 1 \leq i \leq k\right\},
$$

is linearly independent over $\mathbb{Z}$.

Example 3.4 Consider the sum-connectivity Gourava index $\varphi \in \mathbb{R}^{9}$ induced by the symmetric function $\varphi(x, y)=\frac{1}{\sqrt{x+y+x y}}[14]$. Then $\varphi$ is weakly discriminating over $\mathcal{C T}_{n}$. In fact,

$$
\varphi=\left(\frac{\sqrt{5}}{5}, \frac{\sqrt{7}}{7}, \frac{1}{3}, \frac{\sqrt{2}}{4}, \frac{\sqrt{11}}{11}, \frac{\sqrt{2 \times 7}}{14}, \frac{\sqrt{3 \times 5}}{15}, \frac{\sqrt{19}}{19}, \frac{\sqrt{2 \times 3}}{12}\right) .
$$


It follows easily from Besicovitch's Theorem that the set of components of $\varphi$ is a linearly independent set over $\mathbb{Z}$, and by Theorem 3.1, the sum-connectivity Gourava index is weakly discriminating over $\mathcal{C} \mathcal{T}_{n}$.

Based on Besicovitch's Theorem we can introduce weakly discriminating topological indices over arbitrary classes $\mathcal{H} \subseteq \mathcal{G}_{n}$.

Definition 3.5 The prime topological index is defined as follows: let $\mathcal{H} \subseteq \mathcal{G}_{n}, \Delta=$ $\max \{\Delta(H): H \in \mathcal{H}\}$ and $h=\frac{\Delta(\Delta+1)}{2}$. Consider the sequence of the first $h$ prime numbers $\left\{p_{i, j}\right\}_{(i, j) \in K}$. Then we define the VDB topological index $\Pi=\left\{\sqrt{p_{i, j}}\right\}_{(i, j) \in K} \in \mathbb{R}^{h}$.

Clearly, by Besicovitch's Theorem and Theorem 3.1, $\Pi$ is weakly discriminating over $\mathcal{H}$.

Example 3.6 If $\mathcal{H}$ is the set of chemical trees, then $\Pi \in \mathbb{R}^{9}$ is the vector

$$
\Pi=(\sqrt{2}, \sqrt{3}, \sqrt{5}, \sqrt{7}, \sqrt{11}, \sqrt{13}, \sqrt{17}, \sqrt{19}, \sqrt{23}) .
$$

and for a chemical tree $U$,

$$
\begin{aligned}
\Pi(U)= & \sqrt{2} m_{1,2}+\sqrt{3} m_{1,3}+\sqrt{5} m_{1,4}+\sqrt{7} m_{2,2}+ \\
& \sqrt{11} m_{2,3}+\sqrt{13} m_{2,4}+\sqrt{17} m_{3,3}+\sqrt{19} m_{3,4}+\sqrt{23} m_{4,4}
\end{aligned}
$$

We next show how to construct weakly discriminating VDB topological indices $\varphi$ over $\mathcal{H} \subseteq \mathcal{G}_{n}$, when all the components of the vector $\varphi$ are different. First we recall a classical result from algebra. Let $\mathcal{A}$ be the field of algebraic numbers.

Theorem 3.7 [2] (Lindemann-Weierstrass's Theorem) If $\alpha_{1}, \ldots, \alpha_{n}$ are distinct algebraic numbers, then $e^{\alpha_{1}}, \ldots, e^{\alpha_{n}}$ are linearly independent over $\mathcal{A}$.

In [19], the exponential of a VDB topological index $\varphi \in \mathbb{R}^{h}$ over $\mathcal{H} \subseteq \mathcal{G}_{n}$ was defined for $H \in \mathcal{H}$ as

$$
e^{\varphi}(H)=m(H) \cdot e^{\varphi},
$$

where $e^{\varphi} \in \mathbb{R}^{h}$ has components $\left\{e^{\varphi_{i, j}}\right\}_{(i, j) \in K}$.

Theorem 3.8 Let $\varphi \in \mathbb{R}^{h}$ be a VDB topological index defined over $\mathcal{H} \subseteq \mathcal{G}_{n}$ such that all components of $\varphi$ are distinct algebraic numbers. Then $e^{\varphi}$ is weakly discriminating over $\mathcal{H} \subseteq \mathcal{G}_{n}$ 
Proof. Since $\left\{\varphi_{i, j}\right\}_{(i, j) \in K}$ are distinct algebraic numbers, it follows from Theorem 3.7 that $\left\{e^{\varphi_{i, j}}\right\}_{(i, j) \in K}$ are linearly independent over $\mathcal{A}$. But $\mathbb{Z} \subseteq \mathcal{A}$. Hence $\left\{e^{\varphi_{i, j}}\right\}_{(i, j) \in K}$ are linearly independent over $\mathbb{Z}$, and the result follows from Theorem 3.1.

Example 3.9 The components of the Sombor index over $\mathcal{C} \mathcal{T}_{n}$ are distinct algebraic numbers:

$$
S O=(\sqrt{5}, \sqrt{10}, \sqrt{17}, 2 \sqrt{2}, \sqrt{13}, 2 \sqrt{5}, 3 \sqrt{2}, 5,4 \sqrt{2}) .
$$

It follows from Theorem 3.8 that $e^{S O}$ is weakly discriminating over $\mathcal{C} \mathcal{T}_{n}$.

\section{References}

[1] M. Aouchiche, I. El Hallaoui, P. Hansen, Geometric-arithmetic index and minimum degree of connected graphs, MATCH Commun. Math. Comput. Chem. 83 (2020) $179-188$.

[2] A. Baker, Transcendental Number Theory, Cambridge Univ. Press, Cambridge, 1990.

[3] S. Bermudo, J. Nápoles, J. Rada, Extremal trees for the Randić index with given domination number, Appl. Math. Comput. 375 (2020) \#125122.

[4] A. S. Besicovitch, On the linear independence of fractional powers of integers, $J$. London Math. Soc. 15 (1940) 3-6.

[5] R. Cruz, J. Rada, The path and the star as extremal values of vertex-degree-based topological indices among trees, MATCH Commun. Math. Comput. Chem. 82 (2019) $715-732$.

[6] R. Cruz, J. Monsalve, J. Rada, On chemical trees that maximize atom-bond connectivity index, its exponential version, and minimize exponential geometric-arithmetic index, MATCH Commun. Math. Comput. Chem. 84 (2020) 691-718.

[7] R. Cruz, J. Monsalve, J. Rada, Extremal values of vertex-degree-based topological indices of chemical trees, Appl. Math. Comput. 380 (2020) \#125281.

[8] M. Dehmer, M. Grabner, K. Varmuza, Information indices with high discriminative power for graphs, PLoS ONE 7 (2012) \# 31214.

[9] M. Dehmer, M. Grabner, The discrimination power of molecular identification numbers revisited, MATCH Commun. Math. Comput. Chem. 69 (2013) 785-794.

[10] I. Gutman, Degree-based topological indices, Croat. Chem. Acta 86 (2013) 351-361.

[11] I. Gutman, Geometric approach to degree-based topological indices: Sombor indices, MATCH Commun. Math. Comput. Chem. 86 (2021) 11-16. 
[12] I. Gutman, N. Trinajstić, Graph theory and molecular orbitals. Total $\pi$-electron energy of alternant hydrocarbons, Chem. Phys. Lett. 17 (1972) 535-538.

[13] E.V. Konstantinova, On some applications of information indices in chemical graph theory, in: R. Ahlswede, L. Bäumer, N. Cai, H. Aydinian, V. Blinovsky, C. Deppe, H. Mashurian (Eds.), General Theory of Information Transfer and Combinatorics, Springer, Berlin, 2006, pp. 831-852.

[14] V. R. Kulli, On the sum connectivity Gourava index, Int. J. Math. Arch. 8 (2017) 211-217.

[15] V. R. Kulli, Graph indices, in: M. Pal, S. Samanta, A. Pal (Eds.), Handbook of Research of Advanced Applications of Graph Theory in Modern Society, Global, Hershey, 2020, pp. 66-91.

[16] W. Lin, D. Dimitrov, R. Škrekovski, Complete characterization of trees with maximal augmented Zagreb index, MATCH Commun. Math. Comput. Chem. 83 (2020) 167178.

[17] C. T. Martínez-Martínez, J. A. Méndez-Bermúdez, J. M. Rodríguez, J. M. Sigarreta, Computational and analytical studies of the Randić index in Erdős-Rényi models, Appl. Math. Comput. 377 (2020) \#125137.

[18] J. Rada, R. Cruz, Vertex-degree-based topological indices over graphs, MATCH Commun. Math. Comput. Chem. 72 (2014) 603-616.

[19] J. Rada, Exponential vertex-degree-based topological indices and discrimination, MATCH Commun. Math. Comput. Chem. 82 (2019) 29-41.

[20] M. Randić, On characterization of molecular branching, J. Am. Chem. Soc. 97 (1975) 6609-6615.

[21] G. H. Shirdel, H. Rezapour, A. M. Sayadi, The hyper Zagreb index of graph operations, Iran. J. Math. Chem. 4 (2013) 213-220.

[22] R. Todeschini, V. Consonni, Handbook of Molecular Descriptors, Wiley-VCH, Weinheim, 2000.

[23] R. Todeschini, V. Consonni, Molecular Descriptors for Chemoinformatics, WileyVCH, Weinheim, 2009.

[24] R. Zheng, J. Liu, J. Chen, B. Liu, Bounds on the general atom-bond connectivity indices, MATCH Commun. Math. Comput. Chem. 83 (2020) 143-166.

[25] B. Zhou, N. Trinajstić, On a novel connectivity index, J. Math. Chem. 46 (2009) $1252-1270$.

[26] L. Zhong, The harmonic index for graphs, Appl. Math. Lett. 25 (2012) 561-566. 